\title{
Kommunikative Basisaktivitäten im Coaching-Gespräch: Ein linguistischer Beitrag zur Coaching-Prozessforschung
}

\author{
Eva-Maria Graf
}

Online publiziert: 3. Juli 2015

(C) Die Autor(en) 2015. Dieser Artikel ist auf Springerlink.com mit Open Access verfügbar

\begin{abstract}
Zusammenfassung Ausgehend von einem diskursiven Verständnis von Coaching definiert der vorliegende Artikel das Aktivitätenmodell von Coaching als linguistischen Beitrag zur Coaching-Prozessforschung. Im Zentrum dieses Ansatzes steht das Coaching-Gespräch selbst, das mit Hilfe sprachwissenschaftlicher Transkriptionsmethoden verschriftet und mittels einer integrativen Diskursanalyse qualitativ analysiert wird. Der Fokus auf die Gesprächsebene im Coaching erlaubt es, die diskursiv-kommunikativen Besonderheiten von Coaching herauszuarbeiten und so notwendige Einblicke in den Coaching-Prozess zu gewinnen. Es wird argumentiert, dass Coaching von Coach und Klient/in mittels vier kommunikativer Basisaktivitäten ko-konstruiert wird: ,Definieren der Situation', ,Gestalten der Beziehung', ,Ko-konstruieren der Veränderung', ,Evaluieren des Coachings'. Die kommunikative Basisaktivität als Beschreibungs- und Analysekategorien erlaubt es, Fragen nach Coaching-spezifischen diskursiven Praktiken in Abgrenzung zu anderem Beratungshandeln zu klären, aber auch die Bandbreite an beraterischer Interaktion innerhalb von Coaching aufzuzeigen. Ebenso kann mit ihrer Hilfe die Qualität des Coaching-Gesprächs an sich aufgezeigt werden.
\end{abstract}

Schlüsselwörter Coaching-Gespräch · Kommunikative Basisaktivitäten · Diskursive Praktiken · Integrative Diskursanalyse $\cdot$ Coaching-Prozessforschung

\section{Basic Communicative Activities in Coaching: A Linguistic Contribution to the Coaching Process Research}

\begin{abstract}
Based on a discursive understanding of coaching, the current paper presents the basic-activity model of coaching as the linguistic contribution to the coaching process research. Centerpiece of the current approach is the coaching conversation itself, which is transcribed via a linguistic transcription method and analyzed with the help of an integrative discourse analysis. Zooming in on the level of the coaching conversation allows us to distill the discursive and communicative particularities of coaching and thus to gain the necessary insight into the coaching process itself. It will be argued that coaching is co-constructed by coach and client via four basic activities: 'defining the situation', 'building the relationship', 'co-constructing change' and 'evaluating the coaching'. Using 'basic activity' as descriptive and analytic category allows us to answer questions regarding coaching-specific discursive practices in contrast to other consulting or counseling formats; it allows us to display the gamut of coach-client interactions within coaching and, concurrently, the category functions as tool to assess the quality of coaching.
\end{abstract}

Keywords Coaching conversation - Basic communicative activities · Discursive practices - Integrative discourse analysis $\cdot$ Coaching process research

E.-M. Graf $(\bowtie)$

Institut für Anglistik und Amerikanistik,

Alpen-Adria Universität Klagenfurt,

Universitätsstrasse 65-67,

9020 Klagenfurt, Österreich

E-Mail: eva-maria.graf@aau.at 


\section{Prozessforschung im Coaching - Einblicke in die Black Box}

Um den Einfluss und den Beitrag von Executive Coaching und anderer organisationeller Beratungsinterventionen $\mathrm{zu}$ verstehen, ist ein Wissen über ihre generelle Wirksamkeit und ihre Ergebnisse nicht ausreichend. Man muss auch die zugrundeliegenden Coaching-Prozesse selbst - sowohl aus Sicht der Klient/ innen als auch der Coachs - erforschen und sie verstehen lernen (De Haan et al. 2010, S. 11; Übersetzung $\mathrm{EG})$.

Nach einer Phase der Fokussierung auf Fragen der Wirksamkeit von Coaching bzw. relevanter Wirksamkeitsfaktoren im Coaching (vgl. Behrendt 2006; Künzli 2009 oder Greif 2008, 2014) ist in letzter Zeit der Ruf nach (wissenschaftlichen) Einblicken in den Coaching-Prozess selbst lauter geworden (De Haan et al. 2010; Bachkirova et al. 2011; Möller und Kotte 2011). Die reine Ermittlung der Wirksamkeitsfaktoren erlaubt keine Aussagen darüber, wie diese entlang des Coaching-Prozesses in der Interaktion bzw. im Gespräch zwischen Coach und Klient/in umgesetzt oder realisiert werden: „Outcome research has to be silent on what happens within a coaching relationship" (De Haan et al. 2010, S. 110). Gleichzeitig sind Fragen der Wirksamkeit und der Prozessgestaltung aufs engste miteinander verbunden, da die Ermittlung der Prozessfaktoren, die das Ergebnis (positiv) beeinflussen, ein zentrales Anliegen der Prozessforschung darstellt: „Indeed, the ultimate purpose of process research is to make a contribution toward the increased effectiveness of counselling and psychotherapy“" (McLeod 2003, S. 163). Schneider und Kauffeld (2011, S. 190) sprechen in diesem Zusammenhang von einer „neuen Beratungsforschung“, die das Zustandekommen des Endzustands der zwischenmenschlichen Beratungsinteraktion, d. h. das Ergebnis der Berater-Klienten-Interaktion, aus dem Prozess ableitet.

Wie auch die Erforschung der Wirkfaktoren im Coaching ihren Ursprung in der Psychotherapieforschung hat, steht die Coaching-Prozessforschung bezüglich ihrer Annahmen, Fragestellungen und Vorgehensweisen ebenfalls in der Tradition der Psychotherapie-Prozessforschung. Deren Hauptanliegen ist es, die Effekte oder Elemente des Veränderungsprozesses bzw. des Prozesses, der therapeutische Veränderung möglich macht, zu identifizieren, zu beschreiben, $\mathrm{zu}$ erklären und somit auch vorhersagen $\mathrm{zu}$ können (vgl. Greenberg 1986, S. 4). In diesem Zusammenhang unterscheidet Elliott (2010) vier Ansätze innerhalb der change process research $(\mathrm{CPR})$ :

- Quantitatives Prozess-Ergebniss Design

- Qualitatives hilfreiche Faktoren Design
- Mikroanalyse sequentieller Prozesse

- Analyse bedeutsamer Momente

Die linguistische Coaching-Forschung ist durch ihren Fokus auf die Kommunikation bzw. Interaktion im Coaching der Prozessforschung zuzuordnen (vgl. Graf 2008). Möchte man weiterhin den im Zentrum dieses Beitrags stehenden diskursanalytische Ansatz der Kategorisierung der CPR im Sinne Elliotts zuordnen, so ist der linguistische Fokus auf die sprachlich-diskursive Ebene zum einen bei der Analyse bedeutsamer Momente relevant: ein qualitativer Zugang zu den sprachlich-diskursiven Charakteristika von Coaching ermöglicht es, die konkrete, lokale Realisierung dieser bedeutsamen Momente im Hier und Jetzt des jeweiligen Coaching-Gesprächs aufzuzeigen (Graf 2011). Wegener (in Vorb.) argumentiert, dass sich im Zusammenhang mit solchen Momenten die Art und Weise verändert, wie Klient/innen über ihre Themen bzw. Anliegen sprechen. Diese Perspektive auf sich verändernde Thematisierungsmuster in der Sprache der Klient/innen, d. h. wie über etwas gesprochen wird, ist eine wichtige Ergänzung zur rückwirkenden Erschließung bedeutsamer Momente in der Erlebnisperspektive durch die Beteiligten im Rahmen von Interviews. Aus Sicht der Linguistik ist es an dieser Stelle jedoch relevant, noch ein zweites wie in die Analyse zu integrieren und der Frage nachzugehen wie die sich verändernden Thematisierungsmuster in der Sprache der Klient/innen in und durch die Interaktion zwischen Coach und Klient/in ko-konstruiert werden. Im Hinblick auf eine Rückführung der Forschungsergebnisse in die Coaching-Praxis und/oder Coaching-Ausbildung ist ein Bewusstmachen des Spektrums von Verhaltensalternativen, die Coach und Klient/in zur Ko-konstruktion kritischer Momenten zur Verfügung stehen, und eine Sensibilisierung für diese kommunikativen Prozesse (vgl. Lalouschek 1995) von praktischer Relevanz. Das Integrieren der linguistischen Perspektive auf die diskursiv-interaktive Ko-Konstruktion bedeutsamer Momente erfordert eine disziplinen- und methodenübergreifende Herangehensweise; interdisziplinäre Projekte hierzu sind angedacht.

Zum anderen stellt die qualitative Analyse der sprachlichdiskursiven Charakteristika von Coaching ein mikroanalytisch-sequentielles Prozessdesign im Sinne Elliotts (2010) dar. Hierbei ist das Therapie- bzw. Coaching-Gespräch in seinem sequentiellen Entstehen und Charakter primärer Gegenstand der Forschung (siehe hierzu die linguistische Forschung zu (Psycho-)Therapie u. a. von Peräkylä et al. 2008; Pawelczyk 2011; zu AIDS Beratung von Peräkylä 1995 und Silverman 1997 oder zu Berufsberatung von Vehviläinen 1999) und wird mit Hilfe qualitativ-diskursanalytischer Methoden (siehe unten) analysiert. Der Fokus dieses mikroanalytischen Prozessdesigns liegt auf gesamten Sitzungen bzw. Prozessen und auf der Interaktion bzw. dem Gespräch zwischen Therapeut/ in bzw. Coach und Klient/in (,,talk-in-interaction“, Buchholz 
2013, S. 81). In diese Richtung argumentieren auch Beavin et al. (2000, S. 6) mit ihrer „Mikroanalyse der Kommunikation in der Psychotherapie“ " (siehe Bachkirova et al. (2011, S. 11) analog zur Prozessforschung von Coaching):

Communication is not alternating monologues but a tightly interwoven dialogue; these conversations are always collaborative and reciprocally influential. As a result, they are also co-constructive; moment by moment, the therapist and client(s) co-create a version of the problem and its solutions. Because of its centrality, we must examine all therapeutic communication at a micro level, even down to the word or the phrase. In this view, communication is the tool of therapy, just as physical instruments are the tools of surgery, and it is incumbent on us to treat therapeutic communication equally carefully and precisely.

Gleichzeitig aber geht die linguistische Coaching-Forschung - so wie im Folgenden skizziert und verstanden - über eine reine mikroanalytisch-sequentielle CoachingProzessforschung auf textueller Ebene hinaus (vgl. Fairclough 1992). Abschnitt 2 legt die zugrunde liegenden Annahmen des linguistischen Ansatzes dar und erläutert das qualitative Forschungsdesign sowie die wichtigsten Forschungsfragen. Abschnitt 3 präsentiert die Spezifika des Coaching-Gesprächs, ermittelt mit Hilfe des hier nur grob skizzierten linguistischen Coaching-Modells von Coaching. Abschnitt 4 schließlich diskutiert Möglichkeiten und Grenzen dieses Ansatzes und gibt einen Ausblick auf weiterführende Fragestellungen, die mit seiner Hilfe beantwortet werden können.

\section{Sprachwissenschaftliche (Mikro-)Analyse von Coaching-Prozessen}

Die linguistische Erforschung von Coaching-Prozessen ist der qualitativen Sozialforschung zuzuordnen und ist dabei auf der Suche nach ,an understanding of how the world is constructed" (McLeod 2001, S. 2); im vorliegenden Fall auf der Suche nach Antworten auf die Frage, wie Coaching bzw. der Diskurs im und um Coaching konstruiert wird (vgl. Graf 2015a). Gleichzeitig schließt sie durch ihren diskursiven Ansatz auch an die jüngere Organisationsforschung an (vgl. Schulz und Steyaert 2014; Schulz 2013 und diese Ausgabe), die die Bedeutung und kreative Kraft von Sprache bzw. Diskurs im Zusammenhang mit Organisationen bzw. organisationellen Interventionen stärker in den Vordergrund rückt.

\footnotetext{
${ }^{1}$ Allerdings bleibt der Ansatz eine tatsächliche Mikroanalyse therapeutischer Gespräche mit Hilfe gesprächsanalytisch transkribierter authentischer Daten schuldig.
}

Das Hauptaugenmerk des linguistischen Beitrags zur Coaching-Prozessforschung liegt auf den (wiederkehrenden) diskursiven Aktivitäten bzw. Praktiken mittels derer Coach und Klient/in Coaching lokal im Hier und Jetzt ihrer Interaktion ko-konstruieren. Wie auch von Fairclough (1992) argumentiert, bedarf die Analyse und Interpretation dieser diskursiven Praktiken (im Coaching) als den shared habitual practices (Sarangi und Roberts 1999, S. 3; Sarangi 2002), die Coaching - so es sich um einen eigenständigen Beratungstyp handelt - charakterisieren und zu einem eigenständigen Diskurstypen machen, jedoch die Berücksichtigung weiterer diskursiver Ebenen: der konkreten sprachlich-verbalen (und non-verbalen) Ebene im Sinne einer textuellen Mikroperspektive und der sozio-kulturellen, gesellschaftliche Ebenen im Sinne einer diskursiven Makroperspektive zum Zwecke einer kritische Evaluation; auf letztere wird jedoch im Rahmen dieses Beitrags nicht weiter eingegangen (vgl. aber Graf 2015a; Graf und Pawelczyk 2014; Schulz 2013; Schulz und Steyaert 2014). Die analytische Berücksichtigung dieser drei Coaching-Diskursebenen erfordert ein besonderes integratives methodisches Herangehen, das unter Punkt 2.2 kurz erläutert wird.

Der qualitativ-linguistische Fokus auf verschiedene Diskurs-Ebenen im Coaching geschieht im Sinne eines abduktiven Vorgehens dabei weder mit Hilfe a priori präzise definierter theoretischer Konzepte bzw. Kategorien noch rein deduktiv ohne jegliche Vorannahmen aus dem Material heraus. Stattdessen rekonstruiert diese Herangehensweise

... die empirisch begründete Generierung von Konzepten und theoretischen Annahmen als einen Prozess, bei welchem theoretisches Vorwissen mit empirischen [sic] Beobachtungswissen sowohl kreativ als auch methodisch kontrolliert verknüpft werden kann (Kelle und Kluge 2010, S. 13).

Dieses theoretische Vorwissen ermöglicht eine sensibilisierte Wahrnehmung und Interpretation der im Diskurs vorhandenen Phänomene und eine Konkretisierung bzw. Schärfung der Konzepte in Bezug auf den untersuchten Gegenstand, d. h. des Coaching-Diskurses (vgl. Kelle und Kluge 2010). Die hypothetischen Schlussfolgerungen des hier vorgestellten linguistischen Ansatzes basieren - im Sinne des theoretisch Bekannten - auf sprachwissenschaftlichen Erkenntnissen über andere Typen professioneller Interaktion wie Therapie- oder Beratungsgespräch (siehe 2.1), auf theoretischem Coaching-Wissen anderer Disziplinen wie der Organisationspsychologie sowie aus der Coaching-Praxis Literatur. Das Beobachtungswissen entstammt dem empirischen Datenmaterial, d. h. dem Korpus an realen Coaching-Gesprächen, das den Analysen zugrunde liegt (siehe 2.2). 
Coaching aus linguistischer Sicht ist .... Theoretische Vorannahmen

Aus linguistischer Sicht ist Coaching zunächst nicht mehr und nicht weniger als eine bestimmte Art des professionellen Gesprächs, das von den Beteiligten im Hier und Jetzt der Coaching-Sitzung, d. h. lokal, ko-konstruiert wird. Das Gespräch ist dabei sowohl das primäre Medium als auch die primäre Methode im Coaching bzw. das Hauptinstrument, um in der helfenden Interaktion (vgl. Graf und Spranz-Fogasy under review) die professionellen Ziele zu erreichen (vgl. Buchholz 2013). Aufgrund dieses Isomorphismus sollten Coaching-Gespräche im Zentrum der Prozessforschung stehen ${ }^{2}$.

Gespräche zeichnen sich nach Deppermann (2008) aus durch ihre Konstitutivität (Gespräche werden von den Gesprächsteilnehmer/innen aktiv hergestellt), ihre Prozessualität (Gespräche sind zeitliche Gebilde, die durch die Abfolge von Aktivitäten entstehen), ihre Interaktivität (Gespräche bestehen aus wechselseitig aufeinander bezogene Beiträge der Gesprächsteilnehmer/innen), ihre Methodizität (Gesprächsteilnehmer/innen benutzen typische, kulturell verbreitete, d. h. für andere erkennbare und verständliche, Methoden, mit Hilfe derer sie Gesprächsbeiträge konstruieren und interpretieren sowie ihren Austausch unter einander organisieren) und schließlich ihre Pragmatizität (Gesprächsteilnehmer/innen verfolgen im Gespräch teils gemeinsame, teils individuelle Ziele und sie bearbeiten kommunikative Probleme und Aufgaben, die u. a. bei der Organisation des Gesprächs selbst aufkommen).

In Abgrenzung zu Alltagsgesprächen zeichnen sich professionelle Gespräche im allgemeinen und Coaching-Gespräche im Besonderen durch unterschiedliche Beteiligungsvoraussetzungen, Verantwortlichkeiten und Wissensstände der Beteiligten aus (Drew und Heritage 1992; Sator und Graf 2014; Graf und Spranz-Fogasy under review): So eröffnet z. B. in der Regel der Coach die Sitzung oder es werden die Lebensumstände der Klient/innen, nicht aber die des Coachs thematisiert. Im Sinne unterschiedlicher Verantwortlichkeiten geht es um die kommunikativ zu gestaltende Prozesssteuerung, die beim Coach und nicht bei den Klient/innen liegt und bezüglich der Wissens-

\footnotetext{
${ }^{2}$ Analog formuliert Buchholz (2013, S. 82) für die Psychotherapieforschung: „Nicht der Fragebogen, nicht Einschätzungen durch Dritte, nicht ,Messung ' durch Erhebungsinstrumente bilden die Empirie der Psychotherapieforschung, sondern Sprechen und Zuhören, also Konversation, sind allererste Empirie der Psychotherapie. Die anderen empirischen Forschungsmittel erzeugen abgeleitete Empirieformate, oft so, dass man eher Meinung von Beteiligten über Psychotherapie oder Supervision erhebt...Das Primat der Konversation als erste Empirie der Psychotherapie festzustellen, ist somit nichts Geringes.... Konversation. ist primäre empirische Basis aller Arten von Psychotherapie und das schließt Details nicht nur des Verbalen, sondern auch des Mimischen, Stimmlichen und Gestischen selbstverständlich mit ein“.
}

bestände ist anzuführen, dass ,(w)ährend das professionelle Erkenntniswissen spezialisiert, klar umrissen sowie wissenschaftlich fundiert und standardisiert ist ..., ist das Erfahrungswissen der Klient/innen subjektiv, lebensweltlich und individuell“ (Graf und Spranz-Fogasy under review).

,Professionell‘ wird hier als linguistischer terminus technicus verstanden im Sinne einer institutionalisierten, strukturieren Interaktion zwischen dem/der Vertreter/ in einer Profession oder Institution und einem/r Kunde/in oder Klienten/in. Die professionell Agierenden orientieren sich dabei in ihrer Kommunikation mit Klient/innen an den Gesprächen zugrunde liegenden professionellen Agenden, die das Erreichen der professionellen Ziele ermöglichen. Diese Agenden prädeterminieren mehr oder weniger grob den Gesprächsverlauf in seiner internen Aufgaben- und Handlungslogik, wobei die Einhaltung desselben in der Macht der Expert/innen liegt. Asymmetrien und unterschiedliche Macht im Gespräch sind somit ein endemischer Bestandteil professioneller Gespräche.

Zum anderen kann Coaching als Aktivitätstyp definiert werden (vgl. Levinson 1979; Sarangi 2000; Graf 2015a). Unter einem Aktivitätstyp ist eine Gattung oder Kategorie mit fließenden Grenzen zu verstehen, deren Kernmitglieder zielgerichtete, sozial-konstruierte, abgrenzbare interaktive Ereignisse sind mit genauen Vorgaben bezüglich der Teilnehmer/innen, des Settings sowie in besonderem Maße bezüglich der erlaubten Beiträge. Die Teilnehmer/innen interagieren miteinander auf der Basis aktivitätsspezifischer Inferenzen bezüglich der Vorgehensweise, der thematischen Orientierung, der jeweiligen Verantwortlichkeiten etc. $\mathrm{Ob}$ ein bestimmter Beitrag oder eine Handlung Teil eines spezifischen Aktivitätstyps ist, wird von den Beteiligten selbst entlang eines Kontinuums von akzeptablem und nicht-akzeptablem Verhalten eingeordnet (vgl. hierzu das Funktionspendel von Coaching im Sinne des DBVC (2012) sowie die Argumentation von Hall und Duval (2004). Aktivitätstypen bilden sich im Laufe der Zeit aufgrund von Erfahrung rund um einen Kern an Aktivitäten heraus mit spezifischen thematischen, interaktiven und strukturellen Ausprägungen. Die Aktivitäten ihrerseits bestehen aus wiederkehrenden kommunikativen Aufgaben bzw. kommunikativen Zielen und sprachlich-diskursiven Strategien zu ihrer Lösung.

Coaching als professionelles Gespräch und als Aktivitätstyp zu konzeptualisieren, hat folgende Konsequenzen für die sprachwissenschaftliche Analyse bzw. Interpretation von Coaching(-Gesprächen):

1. Das Entstehen von Coaching als zeitliche Abfolge von Aktivitäten erfordert in der Analyse ebenfalls den Blick entlang gesamter Gespräche: der zeitliche Verlauf muss analytisch erfasst werden da z. B. die Beziehung zwischen Coach und Klient/in sich entlang des Prozesses verändert, unterschiedliche Qualitäten entfaltet und mit 
sich verändernden kommunikativen Mitteln realisiert wird. Dieser Veränderung und ihrer lokalen Entstehung bzw. Ko-Konstruktion durch Coach und Klient/in muss analytisch Rechnung getragen werden können. Gespräche allgemein und Coaching-Gespräche im Besonderen als einen zeitlichen Ablauf von Aktivitäten zu deuten erlaubt - im Unterschied zur Fokussieren auf Gesprächsbzw. Prozessphasen - neben der Berücksichtigung dieser strukturellen bzw. zeitlichen Charakteristika auch eine Fokussierung auf thematische und insbesondere interaktive Charakteristika.

2. Da Coaching interaktiv von den Beteiligten ko-konstruiert wird, ist ein einseitiger Fokus auf die Interventionen des Coachs nicht ausreichend, um ihre lokale Funktion und/oder (kommunikative) Wirkung im konkreten Coaching-Gespräch zu bestimmen; stattdessen muss - im Sinne Watzlawick et al's. (1967) punctuation of the sequence of events - berücksichtigt werden, dass Gesprächsbeiträge sowohl die kommunikative Konsequenz des vorher (vom Gegenüber) Gesagten als auch der kommunikative Ko-Text für das danach vom Gegenüber zu Sagende darstellen. Die jeweilige Bedeutung eines Beitrags wird dabei lokal von den Gesprächsteilnehmer/ innen ausgehandelt, das Ergebnis dieser negotiation of meaning (Graf 2007) signalisieren sie sich durch die verbale, non-verbale und paraverbale Gestaltung ihrer Beiträge mit Hilfe von Pausen, Korrekturen, Hörerrückmeldungen etc.

3. Beim Ko-Konstruieren von Gesprächen greifen die Gesprächsteilnehmer/innen auf kulturell-bekannte und etablierte Methoden und Muster in der Gestaltung und Interpretation von Beiträgen zurück. Im Zusammenhang mit Coaching als (noch) nicht standardisiertem und professionalisiertem Beratungsformat bedeutet dies zum einen, dass oftmals nicht auf ein solch kulturelles und etabliertes Wissen zurückgegriffen werden kann. Eine wichtige Frage bei der sprachwissenschaftlichen Analyse von Coaching richtet sich daher an die kommunikativen Konsequenzen dieses Mangels an geteiltem Kontext-Wissen: was bedeutet es für die konkrete kommunikative Interaktion bzw. das Gespräch von Coach und Klient/in? Andererseits sollte sich, sofern Coaching tatsächlich ein eigenständiges Beratungsformat darstellt, die fortschreitende Etablierung, Professionalisierung und damit Standardisierung von Coaching auf kommunikativer Ebene in sich verfestigenden, wiederkehrenden diskursiven Praktiken wiederspiegeln, auf die die Beteiligten zurückgreifen können, die die Aushandlung und Interpretation der kommunikativen Bedeutung der Gesprächsbeiträge von Coach und Klient/in erleichtern und sich in einer Abnahme der Metakommunikation als (noch) konstitutivem Merkmal von Coaching im Sinne Geißler (2013) wiederspiegeln.
4. Im Gespräch verfolgen Teilnehmer/innen teils identische, teils voneinander abweichende Gesprächsziele, die ihre jeweiligen Agenden im Gespräch bestimmen. Anders als bei Alltagsgesprächen orientieren sich bei professionellen Gesprächen wie Coaching die professionell Agierenden, d. h. die Coachs, in ihrer Kommunikation mit Klient/innen an den Gesprächen zugrunde liegenden professionellen Agenden, die das Erreichen ihrer professionellen Ziele (wie z. B. Klärung des Anliegens) ermöglichen. Diese Agenden determinieren mehr oder weniger grob den Gesprächsverlauf in seiner internen Aufgaben- und Handlungslogik, wobei die Einhaltung desselben in der Macht der Expert/innen liegt (und auf sprachlich-diskursiver Ebene u. a. durch sogenannte agenda-moves realisiert wird). Das Erreichen der jeweiligen Gesprächsziele bzw. kommunikativen Ziele ist - im Sinne ihrer diskursiven Etablierung bzw. Ko-konstruktion - die interaktive Voraussetzung für die Coaching-Ziele, die Coach und Klient/in gemeinsam für den jeweiligen Prozess definieren. Das die verschiedenen Interventionen rahmende und realisierende Gespräch ist daher in seiner eigenen diagnostischen und intervenierenden Funktion zu berücksichtigen.

Mit Hilfe der abduktiven Vorgehensweise werden die folgenden Analyse- und Beschreibungskategorien für Coaching sowohl in seiner prozessualen als auch seiner diskursiven Realisierung entwickelt: Coaching entsteht in und durch kommunikative Basisaktivitäten (,basic actitivites“, vgl. Graf 2015a) (vgl. Abschn. 3). Diese kommunikativen Basisaktivitäten setzen sich aus kommunikativen Aufgaben und (wiederkehrenden) diskursiven Praktiken bzw. sprachlichen Verfahren zu deren Lösung zusammen. Die jeweiligen kommunikativen Aufgaben - z. B. die Präsentation des Anliegens ${ }^{3}$ - und ihre sprachlich-kommunikativen Lösungsstrategien sind dabei für den jeweiligen Gesprächstyp konstitutiv (Kallmeyer 1985, 2001). D.h. mit Hilfe der kommunikativen Basisaktivitäten und ihrer konkreten diskursiven Realisierung kann Coaching als eigenständiger Beratungstyp bzw. Diskurstyp etabliert werden (vgl. Graf 2015a). Gleichzeitig ist es über die Ebene der sie konstituierenden kommunikativen Aufgaben möglich, Unterschiede zwischen Coaching-Ansätzen, aber auch zwischen einzelnen Coach-Klient/innen-Dyaden herauszuarbeiten: mit

\footnotetext{
${ }^{3}$ Anliegen zu klären ist eine der Kernaufgaben professioneller Gespräche (siehe z. B. Lalouschek 2008 zu Anliegen im Arzt-Patient-Gespräch oder Pick 2015 zu Anliegensklärung in der Rechtsberatung oder Becker-Mrotzek und Brünner 2007 allgemein-konzeptionell). Die kommunikativen Unterschiede zwischen den verschiedenen Beratungsformaten im Zusammenhang mit der Klärung der Anliegen herauszuarbeiten ist eine zentrale Fragestellung in der linguistischen Erforschung professioneller Gespräche bzw. helfender Interaktionen (vgl. Graf und Spranz-Fogasy under review).
} 
Hilfe von obligatorischen und fakultativen Aufgaben wird das Coaching-Endemische sicht- und analysierbar; idiosynkratische Realisierungsformen können davon unterschieden werden. Die kommunikativen Basisaktivitäten erlauben es auch, die relationale und die thematische Dynamik und Komplexität der Coaching-Interaktion entlang des Prozesses zu beschreiben und zu analysieren.

\section{Methodisches Vorgehen}

Die linguistische Coaching-Prozessforschung erfolgt mit Hilfe einer integrativen Diskursanalyse (Van Dijk 2001; Sarangi 2008; Pawelczyk 2011; Graf 2015a), die eine Beschreibung und Interpretation des Coaching-Prozesses entlang der drei Diskursebenen von Fairclough (1992) sprachlich-verbale bzw. textuellen Ebene, diskursive Praktiken und sozio-kulturelle Praktiken - erlaubt. Bei dieser Methode handelt es sich um ein Integrieren mikroanalytischer Konzepte der Konversations- und Gesprächsanalyse, um Konzepte der interaktionalen Soziolinguistik und Pragmatik für die Erfassung diskursiver Praktiken sowie um markoanalytische Konzepte der kritischen Diskuranalyse, die eine kritische Evaluation von Coaching als sozio-kulturellem Diskurs ermöglichen.

Die Analyse der Coaching-Gespräche - als der diskursiven Ebene, die hier im Zentrum steht - erfolgt entlang des strukturellen, thematischen und interaktiven Verlaufs dieser Gespräche in ihrer Gesamtheit und unter Berücksichtigung aller Beteiligter, als Coach und Klient/in (Pawelczyk 2011; Graf 2015a). Dieses Vorgehen erlaubt es, Fragen nach dem zeitlichen Ablauf von Coaching-Gesprächen, besonders nach sich verändernden kommunikativen Anforderungen an die Beteiligten mit zunehmender Dauer der Interaktion, nach ihrem (sich ebenfalls verändernden) thematischen, und eben auch ihrem (sich verändernden) interaktiven Verlauf zu beantworten.

Als Datenmaterial dienen sogenannte ,authentische“ Coaching-Daten (d. h. Videoaufnahmen von CoachingProzessen bzw. Sitzungen, die nicht zu Forschungs-, Lehroder Illustrationszwecken abgehalten werden), die mit Hilfe der linguistischen Transkriptionsmethode HIAT (halb interpretatives Arbeitstranskript) (vgl. Ehlich und Rehbein 1976) transkribiert wurden. Im Unterscheid zu VerbatimProtokollen, wie sie u. a. für eine qualitative Inhaltsanalyse (Mayring 2010) verwendet werden, liegt das Augenmerk dieser sprachwissenschaftlichen Transkription neben dem Inhalt, dem ,Was' des Gesprächs, vor allem auf der Interaktion selbst, dem ,Wie` des Gesprächs. Zu diesem Zwecke werden Hörersignale, Abbrüche, Überlappungen, Korrekturen, Pausen etc. abgebildet, da sie einen relevanten Einblick in die Interaktion selbst geben und klären helfen, wie gut die Beteiligten in Kontakt sind, wer die Führung im Gespräch für sich beansprucht, wessen Deutung oder Interpretation letztlich übernommen wird etc.

Da die Prozessforschung zu Coaching im Allgemeinen und die linguistische Coaching-Prozessforschung im Besonderen noch in ihren Anfängen stecken, geht es zunächst um eine funktionale Beschreibung des Coaching-Gesprächs: Ist Coaching ein eigenständiger Diskurstyp, der sich aufgrund seiner diskursiven Praktiken von anderen professionellen Diskurstypen unterscheidet? Was genau - wenn - ist dabei das (diskursiv-kommunikativ) Spezifische im CoachingGespräch bzw. was sind Coaching-spezifische diskursive Praktiken? Der folgende Abschnitt gibt dazu Antworten.

\section{Spezifika des Coaching-Gesprächs}

Der Ablauf von Coaching, d. h. der Interaktionsprozess zwischen Coach und Klient/in, wird in der Praxisliteratur zumeist im Rahmen von Phasenmodellen beschrieben, welche postulieren, dass bestimmte Aktivitäten in einer zeitlichen Reihenfolge immer wieder ähnlich ablaufen (vgl. stellvertretend das COACH-Model von Rauen und Steinhübel unter http://www.coaching-report.de/ablauf-des-coachings/ coach-modell.html). Die „sensibilisierte Wahrnehmung“ der Daten (siehe oben) zeigt jedoch, dass ein Phasenmodell bzw. Phasen im Coaching zwar eine hilfreiche Orientierung, jedoch keine hilfreiche Beschreibungs- und Analysekategorie der komplexen Interaktion im Coaching darstellt, um die tatsächlichen und gewünschten Einsichten und Erkenntnisse in die Black Box Coaching zu gewinnen. Als Beispiel sei hier wiederum auf die Beziehungsgestaltung verwiesen, die im Rahmen der Orientierungsphase bei Rauen und Steinhübel (ibid.) folgendermaßen beschrieben wird: „In der Orientierungsphase lernen sich Coach und Klient gegenseitig näher kennen, die Beziehungsklärung schreitet voran und die Beratungsbeziehung wird im Idealfall zunehmend tragfähiger“. Da aber Coaching - wie jede andere Art der (helfenden) Interaktion - erst in und durch die Beziehung der Beteiligten realisiert werden kann, ist es wichtig dieses konstituierende Moment entlang der gesamten Interaktion bzw. des gesamten Prozesses zu berücksichtigen um so seine Coaching-spezifische Ausprägung zu verstehen. Statt mit Hilfe eines Phasenmodells wird der Coaching-Prozess deshalb insgesamt, aber auch das einzelnen Coaching-Gespräch, im Sinne kommunikativer Basisaktivitäten konzeptualisiert und beschrieben (siehe 2.2) .

\footnotetext{
${ }^{4}$ Für die Basisaktivität ,Beziehung gestalten' definiert Graf (2015a) die folgenden kommunikativen Aufgaben: ,Rolle(n) und Identität(en) ,Coach' und ,Klient/in',etablieren', ,Fokus der Coach-Klient/innenBeziehung definieren', ,Hierarchie in asymmetrischer Beziehung aushandeln', ,Coach-Klient/innen Dyade innerhalb des Gefüges Coach-Klient/innen-Organisation ansprechen' sowie ,Beziehung zwischen Coach und Klient/in evaluieren'. Die Aufgaben ihrerseits ent-
} 
Ausgehend von einem solchen Verständnis wurde ein Forschungsprojekt entwickelt, welches der Frage nachgeht, welche zentralen kommunikativen Aktivitäten sich für Coaching identifizieren lassen. Das im Projekt verwendete empirische Material umfasst neun komplette Coaching Prozesse (45 Einzelsitzungen á 2 bis 2,5 S), welche nach der sprachwissenschaftlichen Transkriptionsmethode HIAT verschriftet und dann anonymisiert wurden. Die Coachings wurden von einem weiblichen und einem männlichen (externen) Coach mit insgesamt neun Klient/innen, allesamt Manager/ innen im Versicherungs-, Banken- und Consultingbereich, durchgeführt. Das verwendete Coachingverfahren bezeichnet sich als Emotional-Intelligentes Coaching für Führungskräfte, entwickelt von Dietz Training und Partner.

Eine umfassende Analyse der Daten im Hinblick auf wiederkehrende diskursive Praktiken und ihre sprachliche Realisierung ergab vier (kommunikative) Basisaktivitäten für Coaching (,Definieren der Situation', ,Gestalten der Beziehung ', ,Ko-Konstruieren der Veränderung' und ,Evaluieren des Coachings'), welche im Folgenden erläutert werden:

- ,Definieren der Situation“ beinhaltet all jene kommunikativen Aufgaben, die - zumeist explizit realisiert im Rahmen einer Metakommunikation - den Beteiligten Orientierung geben bezüglich des primären Zwecks und der Art und Weise ihrer Interaktion, d. h. Definieren von Coaching, Erklären der Methode, aber auch Vereinbaren von Terminen oder Integrieren der Erfahrung von Klient/ innen mit Coaching.

- ,Gestalten der Beziehung' beinhaltet die kommunikativen Aufgaben, mittels derer die Beteiligten diskursiv ihre Identitäten und Rollen definieren und dadurch ihre Coaching-Beziehung etablieren und gestalten.

- ,Ko-Konstruieren der Veränderung' beinhaltet die kommunikativen Aufgaben, die das Anliegen und das Ziel definieren und diese gemäß der zugrundeliegenden Coaching-Agenda bearbeiten, um für die Klient/innen Lösungen zu generieren.

- ,Evaluieren des Coachings` beinhaltet die kommunikativen Aufgaben, mittels derer die Beteiligten Coaching thematisch und inhaltlich, relational im Sinne ihrer Beziehung und Zusammenarbeit und prozedural im Sinne des Ablaufs des Coachings evaluieren.

Die vier identifizierten Basisaktivitäten als Beschreibungsund Analysekategorien für Coaching-Interaktion erlauben es eine Beschreibung und Analyse des sequentiellen, thematischen und interaktiven Verlaufs von Coaching sowohl

halten verschiedene Realisierungsmöglichkeiten bzw. Bestandteile: so geschieht z. B. ,Rolle(n) und Identität(en),Coach “ und ,Klient/in“ etablieren' mittels ,Wissen und Expertise', ,Verantwortlichkeiten und Beteiligungsvoraussetzungen', mittels ,Small Talk' und/oder mittels ,persönlicher Information` entlang einzelner Sitzungen als auch entlang des gesamten Prozesses vorzunehmen.

Des Weiteren erlauben die abduktiv entwickelten Kategorien ein Berücksichtigen der komplexen relationalen und diskursiven Dynamik von Coaching, die sich $u$. a. in kommunikativen Schleifen manifestiert. So konnte anhand der analysierten Daten gezeigt werden, dass zwar einzelne Basisaktivitäten einen unterschiedlichen quantitativen Umfang an der gesamten Coaching-Interaktion aufweisen und z. B. ,Ko-Konstruieren der Veränderung' mengenmäBig die umfangreichste Aktivität ist. Des Weiteren erfahren die Kategorien entlang des Prozesses eine unterschiedliche Gewichtung; so widmen sich z. B. Coach und Klient/ in zu Beginn des Coaching-Prozesses in besonderem Maße der Aktivität ,Definieren der Situation', während ,Evaluieren des Coachings' zu Sitzungs- bzw. Prozess-Ende ein verstärktes kommunikatives Engagement der Beteiligten erfährt. Entscheidend ist dabei, dass anhand der Analyse das Spezifische des Coaching-Gesprächs und der Coaching-Prozesse als dynamisches Zusammenspiel zwischen Gewichtung und kommunikativer Ausgestaltung dieser vier Basisaktivitäten beschrieben werden kann. Damit wird auch eine Differenzierung von Coaching gegenüber anderen Beratungsformaten ermöglicht und einzelne Coaching-Ansätze können voneinander unterschieden werden.

Zurückkommend auf den drei Ebenen umfassenden Diskursbegriff von Fairclough (1992), der der hier vorgestellten linguistischen Coaching-Forschung zugrunde liegt, sind die Basisaktivitäten im Sinne seiner (wiederkehrenden) diskursiven Praktiken zu verstehen. Auf dieser „mittleren“"Ebene - die auch im Zentrum dieses Beitrags steht - erhalten wir Einblicke in die generischen Spezifika des Coaching-Gesprächs im Sinne eines eigenen Diskurs- und somit Beratungstyps. Dabei erlauben es die Methode(n) der Diskurs- bzw. Konversationsanalyse auch solche diskursive Praktiken im Coaching offenzulegen, derer sich die Praktiker/innen in dieser Form nicht bewusst sind (vgl. Leudar et al. 2006; Pawelczyk 2011) $)^{5}$.

Fragen nach der konkreten sprachlichen Realisierung - Faircloughs textuelle Ebene - im Sinne eines mikrosequentiellen Fokus erlauben es dagegen, Aussagen über die Qualität bzw. den konkreten Ablauf des Gesprächs ${ }^{6}$ zu tref-

\footnotetext{
${ }^{5}$ Obwohl der Mangel an Professionalisierung und Standardisierung im Coaching allerorts diskutiert wird, finden die konkreten kommunikativen Konsequenzen für Coach und Klient/in im Sinne eines fehlenden kulturellen Kontextwissens kaum Beachtung (aber vgl. Geißlers Konzept der Metakommunikation, Geißler 2013). Die in Graf (2015a) definierte kommunikativen Aufgabe ,Methodisches und Prozedurales Rahmen von Coaching' als obligatorisches Element der Basisaktivität ,Definieren der Situation' trägt diesem Umstand Rechnung und etabliert diese methodische Rahmung als einen festen Bestandteil des Coaching-Gesprächs.

${ }^{6}$ An dieser Stelle sei nochmals auf die Unterscheidung zwischen Gesprächs- und Interventionsebene im Coaching hingewiesen.
} 
fen. Hierbei erfolgt die Interpretation der Gesprächsdaten u. a. in Anlehnung an die Interpretation der Redebeiträge durch die Beteiligten, also Coach und Klient/in, selbst (,display rule“, Sacks et al. 1974): diese signalisieren sich entlang des gesamten Gesprächs gegenseitig durch ihr verbales und paraverbales Verhalten wie Pausen, Reformulierungen, Hörerrückmeldungen etc., wie sie das vom anderen Gesagte interpretieren. Darauf aufbauend lassen sich Aussagen darüber treffen, wie gut die Beteiligten im Kontakt sind, ob oder ob nicht sie gemeinsame Gesprächsziele verfolgen etc. (vgl. Graf 2015b zur linguistischen Evaluierung eines CoachingGesprächs). Zudem kann nur auf dieser konkreten sprachlichen Ebene, also im lokalen (mindestens) drei-gliedrigen Ko-text von Redebeitrag Coach - Redebeitrag Klient/in Redebeitrag Coach (= Follow-up) oder aber Redebeitrag Klient/in - Coach - Klient/in, die spezifische Funktion einer kommunikativen Aktion der Beteiligten (wie etwa einer systemischen Frage durch den Coach) ermittelt werden. So schreibt Schegloff (2007, S. 3), einer der Begründer der amerikanischen Konversationsanalyse, in diesem Zusammehang: ,... sequences of turns are not haphazard but have a shape or structure, and can be tracked for where they came from, what is being done through them, and where they might be going". Als wichtigste Frage, die sich die Beteiligten - und die Diskursanalytiker/innen - in diesem Zusammenhang somit stellen (sollten), lautet, ,warum das (= dieser Beitrag) jetzt?“ (vgl. Schegloff 2007, S. 2).

Auf der Ebene der diskursiven Praktiken und ihrer konkreten sprachlichen Realisierung lassen sich somit sowohl die Spezifika als auch die Qualität von Coaching-Gesprächen - im Sinne eines erfolgreichen Lösens anfallender kommunikativer Aufgaben durch die Beteiligten - linguistisch analysieren.

\section{Ausblick}

Die Grenzen dieses Ansatzes liegen vor allem in der zeitaufwendigen linguistischen Transkriptionsarbeit (1 $\mathrm{min}$ gesprochene Sprache, d. h. Coaching-Gespräch, erfordert minimal 25 min Transkriptionszeit), der nicht-automatisierbaren Analysearbeit und des schwierigen Feldzugangs um noch mehr reale Coaching-Gesprächsdaten zu bekommen. Diesen, vor allem praktischen, Herausforderungen steht jedoch ein großes theoretisch-konzeptionelles wie praktisches Potential des Ansatzes gegenüber, das an dieser Stelle noch einmal abschließend zusammengefasst werden soll:

Als Ergänzung zur existierenden bzw. entstehenden Prozessforschung von Coaching in anderen Disziplinen erlaubt die linguistische Mikro-Analyse verschrifteter Coaching-Gespräche die Berücksichtigung des gesamten Kound Kontexts kritischer bzw. veränderungsrelevanter oder besonders bedeutsamer Momente im Coaching und das
Aufzeigen ihres kommunikativen Entstehens und ermöglicht dadurch ein Sensibilisieren der Beteiligten für kommunikative Prozesse und Verhaltensalternativen im Sinne Lalouscheks (1995).

Das Hauptaugenmerk der linguistischen Coaching-Prozessforschung liegt zum momentanen Zeitpunkt allerdings darauf, die Spezifika und Qualität von Coaching-Gesprächen bzw. Prozessen zu beschreiben und zu analysieren. Bezüglich letzterem ist festzuhalten, dass die Basisaktivitäten bzw. ihre sprachlich-verbale (und non-verbale) Realisierung auf der konkreten Gesprächsebene das Aufzeigen der Qualität des Coaching-Gesprächs anhand der kommunikativen Reaktionen durch die Beteiligten selbst erlauben. Dies ermöglicht - in einem nächsten Schritt - das Formulieren von Handlungsempfehlungen für gelungenes interaktives Gestalten der Basisaktivitäten für praktizierende und lernende Coachs im Rahmen von kommunikativen Sensibilisierungstrainings, Aufbaumodulen etc. im Sinne des von Stein (2008, S. 163) geforderten Tools:

Was, wenn Coachs ein Tool hätten, mit dessen Hilfe sie ihre Gespräche mit Klient/innen hören oder nachlesen könnten und so benennen könnten, was sie in jedem einzelnen Satz oder Phrase, die sie geäußert haben, getan haben? Was, wenn das gleiche Tool Coachs dabei unterstützen könnte, sich jede einzelne Wahl, die sie in ihrem Gespräch mit den Klient/innen treffen, bewusst zu machen? Ein solches Tool würde Coachs in ihrer Selbst-Reflexion als beratend Tätige unterstützen; es würde ihnen ermöglichen, ihre eigenen Gespräche im Moment der Beratung selbst, aber auch danach, zu analysieren um herauszufinden, was funktioniert hat und was nicht. Kurz gesagt, ein solches Tool würde die Erkenntnisse liefern, die Coachs dabei unterstützt, ihre Coaching-Prozesse bestmöglich zu führen (Übersetzung EG).

Das linguistische Coaching-Modell bzw. Aktivitätenmodell mit seinen Analyse- und Beschreibungskategorien der Basisaktivitäten - ,Definieren der Situation', ,Gestalten der Beziehung', ,Ko-Konstruieren der Veränderung' und ,Evaluieren des Coachings " - offenbart vor allem aber wichtige Einblicke in die Black Box Coaching, indem es die Spezifika des Coaching-Gesprächs beschreibbar macht. Mit Hilfe der Basisaktivitäten kann dem prozessualen Charakter von Coaching Rechnung getragen werden, das kommunikative Tun von Coach und Klient/in kann berücksichtigt werden und es ist möglich, die kommunikativ-thematischen Schleifen als relevantem Phänomen des (selbst-)reflexiven Charakters von Coaching zu erfassen. Des Weiteren erlauben sie in ihrem spezifischen Zusammenspiel obligatorischer und fakultativer kommunikativer Aufgaben die Gemeinsamkeiten und Unterschiede des Beratungshandelns im Coaching im Verhältnis zu anderen Formaten zu definie- 
ren sowie unterschiedliche Coaching-Ansätze voneinander abzugrenzen.

Open Access Dieser Artikel wird unter der Creative Commons Namensnennung 4.0 International Lizenz (http://creativecommons. org/licenses/by/4.0/deed.de) veröffentlicht, welche die uneingeschränkte Nutzung, Verbreitung und Wiedergabe für beliebige Zwecke erlaubt, sofern Sie den/die ursprünglichen Autor(en) und die Quelle ordnungsgemäß nennen, einen Link zur Creative Commons Lizenz beifügen und angeben, ob Änderungen vorgenommen wurden.

\section{Literatur}

Bachkirova, T., Sibley, J., \& Myers, A. (2011). Development of an instrument for the microanalysis of coaching sessions. Final progress report. UK: Oxford Brookes University.

Beavin Bavelas, J., McGee, D., Phillips, B., \& Routledge, R. (2000). Microanalysis of communication in psychotherapy. The Journal of Systemic Consultation \& Management, 11(1), 3-22.

Becker-Mrotzek, M., \& Brünner, G. (2007). Anliegensklärung: Anliegen formulieren und explorieren. In A. Redder (Hrsg.), Diskurse und Texte. Festschrift für Konrad Ehlich zum 65. Geburtstag (S. 665-682). Tübingen: Narr.

Behrendt, P. (2006). Wirkung und Wirkfaktoren von psychodramatischem Coaching - eine experimentelle Evaluationsstudie. Zeitschrift für Psychodrama und Soziometrie, 5(1), 59-87.

Buchholz, M. (2013). Die Herausbildung psychotherapeutischer Kompetenz in der Supervision - unterwegs zu Analyse supervisorischer Konversation. In S. Busse \& B. Hausinger (Hrsg.), Supervisionsund Coachingprozesse Erforschen. Theoretische und methodische Zugänge (S. 77-108). Göttingen: Vandenhoeck \& Ruprecht.

De Haan, E., Bertie, C., \& Sills, C. (2010). Critical moments of clients and coaches: A direct-comparison study. International Coaching Psychology Review, 5(2), 109-128.

Deppermann, A. (2008). Gespräche analysieren. Eine Einführung. Wiesbaden: VS Verlag für Sozialwissenschaften.

Deutscher Bundesverband Coaching e. V. (Hrsg.). (2012). Leitlinien und Empfehlungen für die Entwicklung von Coaching als Profession. Osnabrück: Eigenverlag.

Drew, P., \& Heritage, J. (1992). Analyzing talk at work: An introduction. In P. Drew \& J. Heritage (Hrsg.), Talk at work. Interaction in institutional settings (S. 3-65). Cambridge: Cambridge University Press.

Ehlich, K., \& Rehbein, J. (1976). Halbinterpretative Arbeitstranskriptionen (HIAT). Linguistische Berichte, 45, 21-41.

Elliott, R. (2010). Psychotherapy change process research: Realizing the promise. Psychotherapy Research, 20(2), 123-135.

Fairclough, N. (1992). Discourse and social change. Cambridge: Polity Press.

Geißler, H. (2013). Produkt-und Marktsegmentierung von Coaching. In R. Wegener, A. Fritze, \& M. Loebbert (Hrsg.), Coaching-Praxisfelder. Forschung und Praxis im Dialog - Online-Teil (S. 260271). Wiesbaden: Springer.

Graf, E. (2007). „I'm not embarrassed“ - „A little ... uncomfortable? Is that nearer?" The negotiation for meaning in the discourse of coaching. In P. Cap \& J. Nijakowska (Hrsg.), Current trends in pragmatics (S. 281-302). Cambridge: Cambridge Scholar Press.

Graf, E. (2008). Wir kommunizieren also sind wir - Coaching aus kommunikativer und sprachwissenschaftlicher Sicht. Zeitschrift für Organisationsberatung, Supervision, Coaching, 15(2), 156-168.

Graf, E. (2011). Wirksamkeitsforschung und authentische CoachingGesprächsdaten: Ist ,Veränderung' im Coaching mittels sprachwissenschaftlicher Methoden analysierbar? In E. Graf, Y. Aksu,
I. Pick, \& S. Rettinger (Hrsg.), Beratung, Coaching, Supervision: Multidisziplinäre Perspektiven vernetzt (S. 131-146). Wiesbaden: VS-Verlag.

Graf, E. (2015a). The discourses of executive coaching. Linguistic insights into emotionally intelligent coaching. Alpen-Adria Universität Klagenfurt: Habilitationsschrift.

Graf, E. (2015b). Linguistische Evaluation eines Coaching-Prozesses - Die Ko-Konstruktion des Anliegens durch Coach und Klientin. In H. Geißler \& R. Wegener (Hrsg.), Coachingprozesse bewerten. Eine erste Bilanz (S. 211-229). Berlin: Springer.

Graf, E., \& Pawelczyk, J. (2014). The interactional accomplishment of feelings-talk in psychotherapy and executive coaching: Same format, different functions? In E. Graf, M. Sator, \& T. Spranz-Fogasy (Hrsg.), Discourses of helping professions (S. 59-89). Amsterdam: John Benjamins.

Graf, E., \& Spranz-Fogasy, T. (under review). Helfende Berufe - Helfende Interaktionen. In K. Birkner \& N. Janich (Hrsg.), Handbuch Text und Gespräch. Amsterdam: Mouton de Gryter.

Greenberg, L. S. (1986). Change process research. Journal of Consulting and Clinical Psychology, 54, 4-9.

Greif, S. (2008). Coaching und ergebnisorientierte Selbstreflexion. Göttingen: Hogrefe.

Greif, S. (2014). Verbesserung der Qualität und Wirksamkeit im Coaching. In A. Ryba, D. Ginati, D. Pauw, \& R. Stephan (Hrsg.), Coaching im dialog. Weinheim: Beltz (in print).

Hall, M., \& Duval, M. (2004). Meta-coaching. Volume I: Coaching change. For higher levels of success and transformation. Clifton: Neuro-Semantics Publications.

Kallmeyer, W. (1985). Handlungskonstitution im Gespräch: Dupont und sein Experte führen ein Beratungsgespräch durch. In E. Gülich \& T. Kotschi (Hrsg.), Grammatik, Konversation, Interaktion. Beiträge zum Romanistentag 1983 (S. 81-123). Tübingen: Niemeyer.

Kallmeyer, W. (2001). Beraten und Betreuen. Zur gesprächsanalytischen Untersuchung von helfenden Interaktionen. Zeitschrift für Qualitative Bildungs-, Beratungs- und Sozialforschung, 2, 227-252.

Kelle, U., \& Kluge, S. (2010). Vom Einzelfall zum Typus. Fallvergleich und Fallkonstrastierung in der qualitativen Sozialforschung. Wiesbaden: VS Verlag.

Künzli, H. (2009). Wirksamkeitsforschung im Führungskräfte-Coaching. Zeitschrift für Oganisationsberatung, Supervision, Coaching, 16(1), 4-16.

Lalouschek, J. (1995). Ärztliche Gesprächsausbildung. Eine diskursanalytische Studie zu Formen des ärztlichen Gesprächs. Opladen: Westdeutscher Verlag.

Lalouschek, J. (2008). Ärztliche Gesprächspläne und Anliegen von PatientInnen in der chronischen Schmerzbehandlung. Arbeitspapier zum Forschungsprojekt „Schmerzdarstellung und Krankheitserzählung “ (S. 1-65). Universität Wien: Institut für Sprachwissenschaft.

Leudar, I., Antaki, C., \& Barnes, R. (2006). When psychotherapists disclose personal information about themselves to their clients. Communication \& Medicine, 3(1), 27-41.

Levinson, S. (1979). Activity types and language. Linguistics, 17, 356-399.

Mayring, P. (2010). Qualitative Inhaltsanalyse: Grundlagen und Techniken. Weinheim: Beltz.

McLeod, J. (2001). Qualitative research in counselling and psychotherapy. London: Sage Publication.

McLeod, J. (2003). Doing counselling research (2. Aufl.). London: Sage Publication.

Möller, H., \& Kotte, S. (2011). Die Zukunft der Coachingforschung. Zeitschrift für Organisationsberatung, Supervision und Coaching, 18, 445-456.

Pawelczyk, J. (2011). Talk as therapy: Psychotherapy in a linguistic perspective. Amsterdam: Mouton de Gruyter. 
Peräkylä, A. (1995). Aids counselling. Cambridge: Cambridge University Press.

Peräkylä, A., Antaki, Ch, Vehviläinen, S., \& Leudar, I. (Hrsg.). (2008). Conversation analysis of psychotherapy. Cambridge: Cambridge University Press.

Pick, I. (2015). Das anwaltliche Mandantengespräch. Linguistische Ergebnisse zum sprachlichen Handeln von Anwalt und Mandant. Frankfurt/Main: Peter Lang.

Sacks, H., Schegloff, E., \& Jefferson, G. (1974). A simplest systematics for the organisation of turn-taking in conversation. Language, 50(4), 696-735.

Sarangi, S. (2000). Activity types, discourse types and interactional hybridity. In S. Sarangi \& M. Coulthard (Hrsg.), Discourse and social life (S. 1-27). London: Longman.

Sarangi, S. (2002). Discourse practitioners as a community of interprofessional practice: Some insights from health communication research. In C. Candlin (Hrsg.), Research and practice in professional discourse (S. 95-135). Hong Kong: City of University of Hong Kong Press.

Sarangi, S. (2008). Research methods in interprofessional contexts. COMET summer school. Cardiff University.

Sarangi, S., \& Roberts, C. (1999). The dynamics of interactional and institutional orders in work-related settings. In S. Sarangi \& C. Roberts (Hrsg.), Talk, work and institutional order. Discourse in medical, mediation and management settings (S. 1-57). Berlin: Mouton de Gruyter.

Sator, M., \& Graf, E. (2014). ,Making one's path while walking with a clear head" - (Re-)constructing clients' knowledge in the discourse of coaching: Aligning and dis-aligning forms of clients' participation. In E. Graf, M. Sator, \& T. Spranz-Fogasy (Hrsg.), Discourses of helping professions (S. 91-122). Amsterdam: John Benjamins.

Schegloff, E. (2007). Sequence organization in interaction: A primer in conversation Analysis. Cambridge: Cambridge University Press.
Schneider, H., \& Kauffeld, S. (2011). Einflussnahme in Beratungssituationen? Entwicklung des Beobachtungs- und Analyseverfahren act4strategies. In E. Graf, Y. Aksu, I. Pick, \& S. Rettinger (Hrsg.), Beratung, Coaching, Supervision: Multidisziplinäre Perspektiven vernetzt (S. 189-201). Wiesbaden: VS-Verlag.

Schulz, F. (2013). The psycho-managerial complex at work: A study of the discursive practices of management coaching. Dissertation. Switzerland: University of St. Gallen.

Schulz, F. (diese Ausgabe). Ein organisationstheoretischer Ansatz zur Erforschung diskursiver Prozesse im Management Coaching.

Schulz, F., \& Steyaert, C. (2014). Studying Ttlk-at-work. An analysis of the discursive processes of management coaching conversations. In F. Cooren, E. Vaara, A. Langley, \& H. Tsoukas (Hrsg.), Language and communication at work: Discourse, narrativity and organizing (Bd. 4, S. 173-196). Oxford: Oxford University Press.

Silverman, D. (1997). Discourses of counselling. HIV counselling as social interaction. London: Sage.

Stein, I. F. (2008). Enacting the role of coach: Discursive identities in professional coaching discourse. Doctoral dissertation. Fielding Graduate University.

Van Dijk, T. (2001). Multidisciplinary CDA: A plea for diversity. In R. Wodak \& M. Meyer (Hrsg.), Methods of critical discourse analysis (S. 95-120). Los Angeles: Sage Publications.

Vehviläinen, S. (1999). Structures of counselling interaction. A conversation analytic study of counselling encounters in career guidance training. Helsinki: University of Helsinki.

Watzlawick, P., Beavin, J., \& Jackson, D. (1967). Pragmatics of human communication. A study of interactional patterns, pathologies and paradoxes. New York: Norton.

Wegener, R. (in Vorb.). Qualitativ-empirische Rekonstruktion subjektiv bedeutsamer Wirkmomente in Coachingprozessen. 